\title{
Initial time difference quasilinearization for Caputo Fractional Differential Equations
}

\author{
Ali Yakar
}

Correspondence: ali.yakar@gop.edu. tr

Department of Statistics,

Gaziosmanpasa University, Tasliciftlik Campus, 60250 Tokat, Turkey

\begin{abstract}
This paper deals with an application of the method of quasilinearization by not demanding the Hölder continuity assumption of functions involved and by choosing upper and lower solutions with initial time difference for nonlinear Caputo fractional differential equations. Thus, we construct monotone flows that are generated by solutions of linear fractional differential equations which converge uniformly and quadratically to the unique solution of the problem. Also, necessary comparison result concerning lower and upper solutions are proved without using Hölder continuity.
\end{abstract}

Mathematics subject classification: 34A12, 34A45, 34C11.

Keywords: Quasilinearization technique, Caputo fractional differential equation, Quadratic convergence

\section{Introduction}

The method of quasilinearization is employed to provide an explicit analytic representation for the solution of nonlinear differential equations. In this technique, one gets monotone sequences whose iterates are the solutions of corresponding linear problems and furthermore these sequences converge uniformly and quadratically to the unique solution of the given nonlinear differential equations [1]. This is a definite advantage of this constructive technique. Also this method has been generalized, refined and extended in several directions so as to be applicable to a much larger class of nonlinear problems by not demanding convexity or concavity property. Moreover, other possibilities that have been explored make the method of generalized quasilinearization universally useful in applications [2-8].

The concept of noninteger order derivative, popularly known as fractional derivative goes back to the seventeenth century $[9,10]$. Since that time the fractional calculus has drawn the attention of many famous mathematicians. It is only a few decades ago, it was realized that the derivatives of arbitrary order provide an excellent framework for modeling the real world problems in a variety of disciplines. There has been a growing interest in this new area to study the concept of fractional differential equations and fractional dynamical systems [11-18].

The application of quasilinearization for fractional differential equations is a new research area. Depending on development in fractional order differential equations, this technique is reconsidered and similar results parallel to classical theory of

(C) 2012 Yakar; licensee Springer. This is an Open Access article distributed under the terms of the Creative Commons Attribution License (http://creativecommons.org/licenses/by/2.0), which permits unrestricted use, distribution, and reproduction in any medium, provided the original work is properly cited. 
differential equation with integer derivatives have been obtained. Recently, only a few papers were published in this direction [19-21].

We consider the following initial value problem

$$
{ }^{c} D^{q} x(t)=f(t, x), \quad x\left(t_{0}\right)=x_{0}
$$

where $f \in C\left(\left[t_{0}, T\right] \times \mathbb{R}, \mathbb{R}\right)$ and ${ }^{c} D^{q}$ is the Caputo's fractional derivative of order $q$, $0<q<1$.

The corresponding Volterra fractional integral equation is defined as

$$
x(t)=x_{0}+\frac{1}{\Gamma(q)} \int_{t_{0}}^{t}(t-s)^{q-1} f(s, x(s)) \mathrm{d} s
$$

A large cycle of works have been done in the literature using local Hölder continuity assumption which is needed for comparison theorems, see [16,17]. For instance, in [22] the solution of fractional differential equations is obtained by utilizing that condition. Obviously, of great interest is the study of the solution of nonlinear fractional differential equations without using Hölder continuity.

In this work, by not demanding Hölder continuity condition we employe the quasilinearization technique for the given nonlinear fractional order differential equation (1.1) in which upper and lower solutions will have different initial times and positions.

\section{Preliminaries}

In this section, some basic definitions and theorems used throughout the paper are presented. First, we begin with the definition of the class $C_{p}\left[\left[t_{0}, T\right], \mathbb{R}\right]$.

Definition 2.1. A function $\sigma(t)$ is called $a C_{p}$ function if $\sigma \in C\left[\left(t_{0}, T\right], \mathbb{R}\right]$ and $\sigma(t)\left(t-t_{0}\right)^{p} \in C\left[\left[t_{0}, T\right], \mathbb{R}\right]$ with $p=1-q$.

Next, we give the definition of lower and upper solutions, respectively.

Definition 2.2. A function $v \in C_{p}\left[\left[t_{0}, T\right], \mathbb{R}\right], p=1-q, 0<q<1$ is said to be a lower solution of (1.1) if

$$
{ }^{c} D^{q} v(t) \leq f(t, v(t)), \quad v\left(t_{0}\right) \leq x\left(t_{0}\right) .
$$

It is an upper solution if the inequalities are reversed.

Now, consider the following nonhomogeneous linear fractional differential equation,

$$
{ }^{c} D^{q} x=\lambda x+f(t), x\left(t_{0}\right)=x_{0},
$$

where $\lambda$ is a real number and $f \in C_{p}\left(\left[t_{0}, T\right] \times \mathbb{R}, \mathbb{R}\right)$. The equivalent Volterra fractional integral equation for $t_{0} \leq t \leq T$ is

$$
x(t)=x_{0}+\frac{\lambda}{\Gamma(q)} \int_{t_{0}}^{t}(t-s)^{q-1} x(s) \mathrm{d} s+\frac{1}{\Gamma(q)} \int_{t_{0}}^{t}(t-s)^{q-1} f(s) \mathrm{d} s,
$$

When we apply the method of successive approximations (see [16]) to find the solution $x(t)=x\left(t, t_{0}, x_{0}\right)$ explicitly for the given nonhomogeneous IVP (2.1), we obtain

$$
x(t)=x_{0} E_{q}\left(\lambda\left(t-t_{0}\right)^{q}\right)+\int_{t_{0}}^{t}(t-s)^{q-1} E_{q, q}\left(\lambda(t-s)^{q}\right) f(s) \mathrm{d} s, \quad t \in\left[t_{0}, T\right],
$$


where

$$
E_{q}(t)=\sum_{k=0}^{\infty} \frac{t^{k}}{\Gamma(q k+1)} \quad \text { and } \quad E_{q, q}(t)=\sum_{k=0}^{\infty} \frac{t^{k}}{\Gamma(q k+q)}
$$

are Mittag-Leffler functions of one parameter and two parameters, respectively. If $f(t) \equiv 0$, we get, as the solution of the corresponding homogeneous IVP

$$
x(t)=x_{0} E_{q}\left(\lambda\left(t-t_{0}\right)^{q}\right), \quad t \in\left[t_{0}, T\right] .
$$

Remark 2.1. Let ${ }^{c} D^{q} u(t) \leq L u(t), u\left(t_{0}\right)=u_{0}$ where $u \in C_{p}\left(\left[t_{0}, T\right], \mathbb{R}_{+}\right)$and $L$ is positive constant. Then we have the estimate

$$
u(t) \leq u_{0} E_{q}\left(L\left(t-t_{0}\right)^{q}\right) \text { on }\left[t_{0}, T\right]
$$

When $q=1$, that result reduces to well known Gronwall's inequality. For the proof of this remark and further information about Gronwall's type inequality for fractional order differential equations, one can see [23].

If $u_{0}=0$, then $u(t)=0$ identically on $\left[t_{0}, T\right]$.

\section{Comparison Theorem}

In a recent study [24], the Hölder continuity assumption is relaxed to $C_{p}$ continuity of the functions involved in the Riemann-Liouville fractional differential equation. In the following we also prove a comparison result by not requiring the Hölder continuity with a different argument for Caputo fractional differential equations. It is obvious that this result is essential to extend the applicability of iterative techniques such as the monotone iterative technique and the method of quasilinearization.

Theorem 3.1. Let $v(t), w(t) \in C_{p}\left[\left[t_{0}, T\right], \mathbb{R}\right]$ and $f \in C\left[\left[t_{0}, T\right] \times \mathbb{R}, \mathbb{R}\right]$ and

$$
\begin{aligned}
\text { (i) }{ }^{c} D^{q} v(t) & \leq f(t, v(t)) \\
\text { (ii) }{ }^{c} D^{q} w(t) & \geq f(t, w(t))
\end{aligned}
$$

Suppose further that the standard Lipschitz condition is satisfied

$$
f(t, x)-f(t, y) \leq L(x-y), \quad x \geq y
$$

and $L>0$.

Then $v\left(t_{0}\right) \leq w\left(t_{0}\right)$ implies

$$
v(t) \leq w(t), \quad t_{0} \leq t \leq T .
$$

Proof. Suppose that $v(t) \leq w(t)$ for $t_{0} \leq t \leq T$ is not true. Then, there exists a $t_{1}>t_{0}$ such that $v\left(t_{1}\right)>w\left(t_{1}\right)$ and $t_{0}<t_{1} \leq T$. Since $v\left(t_{0}\right) \leq w\left(t_{0}\right)$ we encounter with two cases in view of the continuity of functions involved:

(i) If $v\left(t_{0}\right)<w\left(t_{0}\right)$, then one can find a $\tau_{0}$ such that $v\left(\tau_{0}\right)=w\left(\tau_{0}\right)$ and $t_{0}<\tau_{0}<t_{1}$. Thus, we have $v(t)>w(t)$ on $\left(\tau_{0}, t_{1}\right]$.

(ii) If $v\left(t_{0}\right)=w\left(t_{0}\right)$, then two situations are possible. Namely, one can get $v(t)>w(t)$ on $\left(t_{0}, t_{1}\right]$ or $v(t)>w(t)$ on $\left(\tau_{0}, t_{1}\right]$ where $t_{0}<\tau_{0}<t_{1}$ and $v\left(\tau_{0}\right)=w\left(\tau_{0}\right)$ as before.

In both cases, we can find an interval $\left[t_{0}, t_{1}\right]$ or $\left[\tau_{0}, t_{1}\right]$ on which $v(t) \geq w(t)$.

Let us define

$$
u(t)=v(t)-w(t)
$$


and assume that $u(t)$ is defined on $\left[\tau_{0}, t_{1}\right]$ (or on $\left[t_{0}, t_{1}\right]$ ). Note that $u(t)>0$ on $\left(\tau_{0}, t_{1}\right]$ and $u\left(\tau_{0}\right)=0$. Taking Caputo's fractional derivative of both sides of (3.3), we get

$$
{ }^{c} D^{q} u(t)={ }^{c} D^{q} v(t)-{ }^{c} D^{q} w(t)
$$

By using the lower and upper properties of $v(t)$ and $w(t)$, we have

$$
{ }^{c} D^{q} u(t) \leq f(t, v(t))-f(t, w(t))
$$

Since $f$ is Lipschitz with $L>0$ and $v(t) \geq w(t)$ on $\left[\tau_{0}, t_{1}\right]$, we obtain

$$
{ }^{c} D^{q} u(t) \leq L \cdot[v(t)-w(t)],
$$

Therefore we get

$$
{ }^{c} D^{q} u(t) \leq L u(t), \quad u\left(\tau_{0}\right)=0,
$$

which implies, in view of Remark 2.1,

$$
u(t)=0 \quad \text { for } \quad \tau_{0} \leq t \leq t_{1}
$$

which gives a contradiction. So, we have $v(t) \leq w(t)$ on $\left[t_{0}, T\right]$.

Corollary 3.1 The function $f(t, u)=\sigma(t) u$, where $\sigma(t) \leq L$ is admissible in Theorem 3.1 to yield $u(t) \leq 0$ on $t_{0} \leq t \leq T$.

Observe that a dual result of corollary 2.1 is valid.

\section{Quasilinearization with Initial Time Difference}

The purpose of this section is to employ the quasilinearization technique for nonlinear Caputo's fractional order differential equation (1.1) by choosing lower and upper solutions with initial time difference and not imposing the Hölder continuity on functions involved. Also, we consider the function $f(t, x)$ on the right hand side of the equation (1.1) which satisfies a weaker condition than convexity.

Theorem 4.1. Assume that

(i) $\alpha \in C_{p}\left[\left[t_{0}, t_{0}+T\right], \mathbb{R}\right], t_{0}, T>0, \beta \in C_{p}\left[\left[\tau_{0}, \tau_{0}+T\right], \mathbb{R}\right], \tau_{0}>0, f \in C\left[\left[t_{0}, \tau_{0}+T\right] \times\right.$ $\mathbb{R}, \mathbb{R}]$ and

$$
\begin{array}{ll}
{ }^{c} D^{q} \alpha(t) & \leq f(t, \alpha(t)), \quad t_{0} \leq t \leq t_{0}+T \\
{ }^{c} D^{q} \beta(t) & \geq f(t, \beta(t)), \quad \tau_{0} \leq t \leq \tau_{0}+T
\end{array}
$$

with $\alpha\left(t_{0}\right) \leq x\left(s_{0}\right) \leq \beta\left(\tau_{0}\right)$ and $t_{0}<s_{0}<\tau_{0}$ where $\alpha(t) \leq \beta\left(t+\eta_{1}\right), t_{0} \leq t \leq t_{0}+T$ and $\eta_{1}=\tau_{0}-t_{0}$;

(ii) Suppose $f_{x}(t, x)$ exists and following relations hold

$$
\begin{gathered}
f(t, x) \geq f(t, y)+f_{x}(t, y)(x-y) \quad \text { whenever } x \geq y \text { and } \\
\left|f_{x}(t, x)-f_{x}(t \cdot y)\right| \leq L|x-y|, \quad L>0 ;
\end{gathered}
$$

(iii) $f(t, x)$ is nondecreasing in $t$ for each $x$ and $f_{x}(t, x)$ is nondecreasing in $x$ for each $t$.

Then there exists monotone sequences $\left\{\widetilde{\alpha}_{n}\right\}$ and $\left\{\widetilde{\beta}_{n}\right\}$ which converge uniformly and monotonically to the unique solution of (1.1) with $x\left(s_{0}\right)=x_{0}$ on $\left[s_{0}, s_{0}+T\right]$ and the convergence is quadratic. 
Proof. Let $\widetilde{\beta}_{0}(t)=\beta\left(t+\eta_{1}\right)$ and $\widetilde{\alpha}_{0}(t)=\alpha(t), t_{0} \leq t \leq t_{0}+T$ where $\eta_{1}=\tau_{0}-t_{0}$. Then we have

$$
\widetilde{\beta}_{0}\left(t_{0}\right)=\beta\left(\tau_{0}\right) \geq \alpha\left(t_{0}\right)=\widetilde{\alpha}\left(t_{0}\right) .
$$

Also

$$
\begin{aligned}
{ }^{c} D^{q} \tilde{\beta}_{0}(t) & ={ }^{c} D^{q} \beta\left(t+\eta_{1}\right) \\
& \geq f\left(t+\eta_{1}, \beta\left(t+\eta_{1}\right)\right) \\
& =f\left(t+\eta_{1}, \tilde{\beta}_{0}(t)\right)
\end{aligned}
$$

since $f(t, x)$ is nondecreasing in $t$ for each $x$, we get

$$
{ }^{c} D^{q} \tilde{\beta}_{0}(t) \geq f\left(t, \tilde{\beta}_{0}(t)\right)
$$

Similarly, we can write

$$
{ }^{c} D^{q} \tilde{\alpha}_{0}(t)={ }^{c} D^{q} \alpha_{0}(t) \leq f\left(t, \alpha_{0}(t)\right)=f\left(t, \tilde{\alpha}_{0}(t)\right)
$$

which shows $\tilde{\alpha}_{0}(t)$ is a lower solution of the problem.

Consider the following linear fractional equations

$$
\begin{array}{ll}
{ }^{c} D^{q} \tilde{\alpha}_{n+1}(t)=f\left(t+\eta_{2}, \tilde{\alpha}_{n}\right)+f_{x}\left(t+\eta_{2}, \tilde{\alpha}_{n}\right)\left(\tilde{\alpha}_{n+1}-\tilde{\alpha}_{n}\right), & \widetilde{\alpha}_{n+1}\left(t_{0}\right)=x_{0} \\
{ }^{c} D^{q} \widetilde{\beta}_{n+1}(t)=f\left(t+\eta_{2}, \widetilde{\beta}_{n}\right)+f_{x}\left(t+\eta_{2}, \widetilde{\alpha}_{n}\right)\left(\widetilde{\beta}_{n+1}-\widetilde{\beta}_{n}\right), & \widetilde{\beta}_{n+1}\left(t_{0}\right)=x_{0}
\end{array}
$$

where $\eta_{2}=s_{0}-t_{0}$. Note that unique solutions exist since the right hand side of the equations satisfy a Lipschitz condition.

We shall show that

$$
\widetilde{\alpha}_{0} \leq \widetilde{\alpha}_{1} \leq \cdots \leq \widetilde{\alpha}_{n} \leq \widetilde{\beta}_{n} \leq \cdots \leq \widetilde{\beta}_{1} \leq \widetilde{\beta}_{0} \text { on }\left[t_{0}, t_{0}+T\right] .
$$

First we must prove

$$
\widetilde{\alpha}_{0} \leq \widetilde{\alpha}_{1} \leq \widetilde{\beta}_{1} \leq \widetilde{\beta}_{0} \quad \text { on }\left[t_{0}, t_{0}+T\right] .
$$

Set $p(t)=\widetilde{\alpha}_{1}-\widetilde{\alpha}_{0}$ then

$$
\begin{aligned}
{ }^{c} D^{q} p(t) & ={ }^{c} D^{q} \widetilde{\alpha}_{1}-{ }^{c} D^{q} \widetilde{\alpha}_{0} \\
& \geq f\left(t+\eta_{2}, \widetilde{\alpha}_{0}\right)+f_{x}\left(t+\eta_{2}, \widetilde{\alpha}_{0}\right)\left(\widetilde{\alpha}_{1}-\widetilde{\alpha}_{0}\right)-f\left(t+\eta_{2}, \widetilde{\alpha}_{0}\right) \\
& =f_{x}\left(t+\eta_{2}, \widetilde{\alpha}_{0}\right)\left(\widetilde{\alpha}_{1}-\widetilde{\alpha}_{0}\right) \\
{ }^{c} D^{q} p(t) & \geq f_{x}\left(t+\eta_{2}, \widetilde{\alpha}_{0}\right) p, p\left(t_{0}\right) \geq 0 .
\end{aligned}
$$

Hence applying Corollary 3.1 , we get $\widetilde{\alpha}_{0} \leq \widetilde{\alpha}_{1}$ on $\left[t_{0}, t_{0}+T\right]$. Similarly, one can show that $\widetilde{\beta}_{1} \leq \widetilde{\beta}_{0}$.

Now we must prove that $\widetilde{\alpha}_{1} \leq \tilde{\beta}_{1}$ on $\left[t_{0}, t_{0}+T\right]$. To do so, we set $p(t)=\widetilde{\beta}_{1}(t)-\widetilde{\alpha}_{1}(t)$, then

$$
\begin{aligned}
{ }^{c} D^{q} p(t)= & { }^{c} D^{q} \widetilde{\beta}_{1}-{ }^{c} D^{q} \widetilde{\alpha}_{1} \\
= & f\left(t+\eta_{2}, \widetilde{\beta}_{0}\right)+f_{x}\left(t+\eta_{2}, \widetilde{\alpha}_{0}\right)\left(\widetilde{\beta}_{1}-\widetilde{\beta}_{0}\right) \\
& -\left[f\left(t+\eta_{2}, \widetilde{\alpha}_{0}\right)+f_{x}\left(t+\eta_{2}, \widetilde{\alpha}_{0}\right)\left(\widetilde{\alpha}_{1}-\widetilde{\alpha}_{0}\right)\right] \\
= & f\left(t+\eta_{2}, \widetilde{\beta}_{0}\right)-f\left(t+\eta_{2}, \widetilde{\alpha}_{0}\right)+f_{x}\left(t+\eta_{2}, \widetilde{\alpha}_{0}\right)\left(\widetilde{\beta}_{1}-\widetilde{\beta}_{0}-\widetilde{\alpha}_{1}+\widetilde{\alpha}_{0}\right)
\end{aligned}
$$


using the inequality in (ii) we get

$$
\begin{aligned}
{ }^{c} D^{q} p(t) & \geq f_{x}\left(t+\eta_{2}, \widetilde{\alpha}_{0}\right)\left(\widetilde{\beta}_{0}-\widetilde{\alpha}_{0}\right)+f_{x}\left(t+\eta_{2}, \widetilde{\alpha}_{0}\right)\left(\widetilde{\beta}_{1}-\widetilde{\beta}_{0}-\widetilde{\alpha}_{1}+\widetilde{\alpha}_{0}\right) \\
& \geq f_{x}\left(t+\eta_{2}, \widetilde{\alpha}_{0}\right)\left(\widetilde{\beta}_{1}-\widetilde{\alpha}_{1}\right)
\end{aligned}
$$

this implies that

$$
{ }^{c} D^{q} p(t) \geq f_{x}\left(t+\eta_{2}, \tilde{\alpha}_{0}\right) p(t) \text { and } p\left(t_{0}\right)=0
$$

which because of Corollary 3.1 yields $p(t) \geq 0$. Thus we have $\widetilde{\alpha}_{1} \leq \widetilde{\beta}_{1}$ on $\left[t_{0}, t_{0}+\right.$ $T]$. Hence (4.4) is proved.

Using mathematical induction with $k>1$, we obtain

$$
\widetilde{\alpha}_{0} \leq \widetilde{\alpha}_{k-1} \leq \widetilde{\alpha}_{k} \leq \widetilde{\beta}_{k} \leq \widetilde{\beta}_{k-1} \leq \widetilde{\beta}_{0} \quad \text { on }\left[t_{0}, t_{0}+T\right] .
$$

Now we need to show that

$$
\widetilde{\alpha}_{k} \leq \widetilde{\alpha}_{k+1} \leq \widetilde{\beta}_{k+1} \leq \widetilde{\beta}_{k} \quad \text { on }\left[t_{0}, t_{0}+T\right] .
$$

To prove this, we set $p(t)=\widetilde{\alpha}_{k+1}-\widetilde{\alpha}_{k}$ so that utilizing equations in (4.1), (4.2) and the inequality in (ii), we have

$$
\begin{aligned}
{ }^{c} D^{q} p(t)= & f\left(t+\eta_{2}, \widetilde{\alpha}_{k}\right)+f_{x}\left(t+\eta_{2}, \widetilde{\alpha}_{k}\right)\left(\widetilde{\alpha}_{k+1}-\widetilde{\alpha}_{k}\right) \\
& -\left[f\left(t+\eta_{2}, \widetilde{\alpha}_{k-1}\right)+f_{x}\left(t+\eta_{2}, \widetilde{\alpha}_{k-1}\right)\left(\widetilde{\alpha}_{k}-\widetilde{\alpha}_{k-1}\right)\right] \\
\geq & f_{x}\left(t+\eta_{2}, \widetilde{\alpha}_{k-1}\right)\left(\widetilde{\alpha}_{k}-\widetilde{\alpha}_{k-1}\right)+f_{x}\left(t+\eta_{2}, \widetilde{\alpha}_{k}\right)\left(\widetilde{\alpha}_{k+1}-\widetilde{\alpha}_{k}\right) \\
& -f_{x}\left(t+\eta_{2}, \widetilde{\alpha}_{k-1}\right)\left(\widetilde{\alpha}_{k}-\widetilde{\alpha}_{k-1}\right) \\
\geq & f_{x}\left(t+\eta_{2}, \widetilde{\alpha}_{k}\right)\left(\widetilde{\alpha}_{k+1}-\widetilde{\alpha}_{k}\right) .
\end{aligned}
$$

Thus we obtain

$$
{ }^{c} D^{q} p(t) \geq f_{x}\left(t+\eta_{2}, \widetilde{\alpha}_{k}\right) p(t) \quad \text { and } p\left(t_{0}\right)=0 .
$$

Again using corollary 3.1 we get $\widetilde{\alpha}_{k} \leq \widetilde{\alpha}_{k+1}$ on $\left[t_{0}, t_{0}+T\right]$. In a similar way, it can be shown that $\widetilde{\beta}_{k} \geq \widetilde{\beta}_{k+1}$ on $\left[t_{0}, t_{0}+T\right]$. Next we must prove $\widetilde{\alpha}_{k+1} \leq \widetilde{\beta}_{k+1}$ on $\left[t_{0}, t_{0}+T\right]$. Let $p(t)=\widetilde{\beta}_{k+1}-\widetilde{\alpha}_{k+1}$, then

$$
\begin{aligned}
{ }^{c} D^{q} p(t)= & { }^{c} D^{q} \widetilde{\beta}_{k+1}-{ }^{c} D^{q} \widetilde{\alpha}_{k+1} \\
= & f\left(t+\eta_{2}, \widetilde{\beta}_{k}\right)+f_{x}\left(t+\eta_{2}, \widetilde{\alpha}_{k}\right)\left(\widetilde{\beta}_{k+1}-\widetilde{\beta}_{k}\right) \\
& -\left[f\left(t+\eta_{2}, \widetilde{\alpha}_{k}\right)+f_{x}\left(t+\eta_{2}, \widetilde{\alpha}_{k}\right)\left(\widetilde{\alpha}_{k+1}-\widetilde{\alpha}_{k}\right)\right] \\
\geq & f_{x}\left(t+\eta_{2}, \widetilde{\alpha}_{k}\right)\left(\widetilde{\beta}_{k}-\widetilde{\alpha}_{k}\right)+f_{x}\left(t+\eta_{2}, \widetilde{\alpha}_{k}\right)\left(\widetilde{\beta}_{k+1}-\widetilde{\beta}_{k}\right) \\
& -f_{x}\left(t+\eta_{2}, \widetilde{\alpha}_{k}\right)\left(\widetilde{\alpha}_{k+1}-\widetilde{\alpha}_{k}\right) \\
= & f_{x}\left(t+\eta_{2}, \widetilde{\alpha}_{k}\right)\left[\widetilde{\beta}_{k}-\widetilde{\alpha}_{k}-\widetilde{\alpha}_{k+1}+\widetilde{\alpha}_{k}+\widetilde{\beta}_{k+1}-\widetilde{\beta}_{k}\right] \\
\geq & f_{x}\left(t+\eta_{2}, \widetilde{\alpha}_{k}\right)\left(\widetilde{\beta}_{k+1}-\widetilde{\alpha}_{k+1}\right)
\end{aligned}
$$

Thus we have ${ }^{c} D^{q} p(t) \geq f_{x}\left(t+\eta_{2}, \widetilde{\alpha}_{k}\right) p(t)$ and $p\left(t_{0}\right)=0$. It follows from the Corollary 3.1 we reach $\widetilde{\alpha}_{k+1} \leq \widetilde{\beta}_{k+1}$ on $\left[t_{0}, t_{0}+T\right]$. Hence (4.6) is proved.

Employing standard techniques (see [16]), it can be easily shown that the monotone sequences $\left\{\widetilde{\alpha}_{n}\right\}$ and $\left\{\widetilde{\beta}_{n}\right\}$ converge uniformly and monotonically to the unique solution $\tilde{x}(t)$ of 


$$
{ }^{c} D^{q} \tilde{x}(t)=f\left(t+\eta_{2}, \tilde{x}(t)\right), \quad \tilde{x}\left(t_{0}\right)=x_{0}
$$

Letting $s=t+\eta_{2}$ and changing the variable, we have

$$
{ }^{c} D^{q} x=f(s, x), \quad x\left(s_{0}\right)=x_{0} .
$$

Next we will prove that the convergence is quadratic. For this purpose, consider

$$
p_{n+1}=\tilde{x}-\tilde{\alpha}_{n+1}
$$

Note that $p_{n+1}\left(t_{0}\right)=0$. So we have

$$
\begin{aligned}
{ }^{c} D^{q} p_{n+1} & ={ }^{c} D^{q} \tilde{x}-{ }^{c} D^{q} \widetilde{\alpha}_{n+1} \\
& =f\left(t+\eta_{2}, \widetilde{x}\right)-\left[f\left(t+\eta_{2}, \widetilde{\alpha}_{n}\right)+f_{x}\left(t+\eta_{2}, \widetilde{\alpha}_{n}\right)\left(\widetilde{\alpha}_{n+1}-\widetilde{\alpha}_{n}\right)\right] \\
& =\left[f\left(t+\eta_{2}, \widetilde{x}\right)-f\left(t+\eta_{2}, \widetilde{\alpha}_{n}\right)\right]-f_{x}\left(t+\eta_{2}, \widetilde{\alpha}_{n}\right)\left(p_{n}-p_{n+1}\right) \\
& \leq\left[f_{x}\left(t+\eta_{2}, \widetilde{x}\right)-f_{x}\left(t+\eta_{2}, \widetilde{\alpha}_{n}\right)\right] p_{n}+f_{x}\left(t+\eta_{2}, \widetilde{\alpha}_{n}\right) p_{n+1} \\
& \leq L\left|p_{n}\right|^{2}+f_{x}\left(t+\eta_{2}, \widetilde{\alpha}_{n}\right) p_{n+1} \\
& \leq L\left|p_{n}\right|_{0}^{2}+N \cdot p_{n+1}
\end{aligned}
$$

where $\left|f_{x}\right| \leq N,\left|p_{n}\right|_{0}=\max _{\left[t_{0}, t_{0}+T\right]}\left|p_{n}(t)\right|$. This inequality gives the estimate

$$
\begin{aligned}
p_{n+1} & \leq L\left|p_{n}\right|_{0}^{2} \int_{t_{0}}^{t}(t-s)^{q-1} E_{q, q}\left(N(t-s)^{q}\right) \mathrm{d} s \\
& \leq N_{0}\left|p_{n}\right|_{0}^{2}
\end{aligned}
$$

where $N_{0}=\frac{L T^{q}}{q} E_{q, q}\left(N T^{q}\right)$ and $E_{q, q}$ is Mittag-Leffler function.

Thus we reach the desired result

$$
\max _{\left[t_{0}, t_{0}+T\right]}\left|\widetilde{x}-\widetilde{\alpha}_{n+1}\right| \leq N_{0} \max _{\left[t_{0}, t_{0}+T\right]}\left|\tilde{x}-\tilde{\alpha}_{n}\right|^{2} .
$$

Similarly, after using suitable computation, we get the quadratic convergence of $\left\{\widetilde{\beta}_{n}\right\}$ such that

$$
\max _{\left[t_{0}, t_{0}+T\right]}\left|\tilde{\beta}_{n+1}-\tilde{x}\right| \leq \frac{N_{0} L}{2} \max _{\left[t_{0}, t_{0}+T\right]}\left|\tilde{x}-\tilde{\alpha}_{n}\right|^{2}+\frac{3 N_{0} L}{2} \max _{\left[t_{0}, t_{0}+T\right]}\left|\tilde{\beta}_{n}-\tilde{x}\right|^{2} .
$$

The proof is complete.

Corollary 4.1. If the assumptions of Theorem 4.1 hold with $s_{0}=t_{0}$, then the conclusion remains the same.

Proof. For the proof, we let $\tilde{\beta}_{0}(t)=\beta\left(t+\eta_{1}\right), \tilde{\alpha}_{0}(t)=\alpha(t)$, and $\tilde{x}(t)=x(t)$ on $\left[t_{0}, t_{0}+\right.$ $T]$ and we proceed as we did in Theorem 4.1.

Corollary 4.2. If the assumptions of Theorem 4.1 hold with $s_{0}=\tau_{0}$, then the conclusion remains the same.

Proof. This time, we must set $\tilde{\alpha}_{0}(t)=\alpha\left(t-\eta_{1}\right), \tilde{\beta}_{0}(t)=\beta(t)$ and $\tilde{x}(t)=x(t)$ on $\left[\tau_{0}\right.$, $\left.\tau_{0}+T\right]$ proceed as we did in Theorem 4.1.

In case $t_{0}>\tau_{0}$, a dual result of Theorem 4.1 can be proved with some suitable changes. Next result is given in this direction.

Theorem 4.2. Assume that 
(i) $\alpha \in C_{p}\left[\left[t_{0}, t_{0}+T\right], \mathbb{R}\right], t_{0}, T>0, \beta \in C_{p}\left[\left[\tau_{0}, \tau_{0}+T\right], \mathbb{R}\right], \tau_{0}>0, f \in C\left[\left[\tau_{0}, t_{0}+T\right] \times\right.$ $\mathbb{R}, \mathbb{R}]$ and

$$
\begin{array}{ll}
{ }^{c} D^{q} \alpha(t) & \leq f(t, \alpha(t)), \quad t_{0} \leq t \leq t_{0}+T \\
{ }^{c} D^{q} \beta(t) & \geq f(t, \beta(t)), \quad \tau_{0} \leq t \leq \tau_{0}+T
\end{array}
$$

with $\alpha\left(t_{0}\right) \leq x\left(s_{0}\right) \leq \beta\left(\tau_{0}\right)$ and $\tau_{0}<s_{0}<t_{0}$ where $\alpha\left(t+\eta_{1}\right) \leq \beta(t), \tau_{0} \leq t \leq \tau_{0}+T$ and $\eta_{1}=t_{0}-\tau_{0}$

(ii) Suppose $f_{x}(t, x)$ exists and following relations hold

$$
\begin{gathered}
f(t, x) \geq f(t, y)+f_{x}(t, y)(x-y) \quad \text { whenever } x \geq y \text { and } \\
\left|f_{x}(t, x)-f_{x}(t . y)\right| \leq L|x-y|, \quad L>0
\end{gathered}
$$

(iii) $f(t, x)$ is nonincreasing in $t$ for each $x$ and $f_{x}(t, x)$ is nondecreasing in $x$ for each $t$. Then the conclusion of theorem 4.1 remains valid.

Proof. The proof being similar to theorem 4.1, we omit details.

\section{Conclusion}

In this work, the quasilinearization technique coupled with lower and upper solutions is employed to study Caputo fractional differential equations. We have observed that this technique is convenient even though initial functions $\alpha$ and $\beta$ are given with initial times. In this way, by not requiring Hölder continuity condition, one gets monotone sequences whose iterates are solutions of corresponding linear problems and the sequences converge uniformly and quadratically to the unique solution of the given nonlinear problem.

\section{Acknowledgements}

This work has been supported by The Scientific and Technological Research Council of Turkey (TÜBITAK).

\section{Competing interests}

The author declares that they have no competing interests.

Received: 30 July 2011 Accepted: 27 June 2012 Published: 27 June 2012

\section{References}

1. Lakshmikantham, V, Vatsala, AS: Generalized Quasilinearization for Nonlinear Problems. Kluwer Academic Publisher, The Netherlands (1998)

2. Köksal, S, Yakar, C: Generalized quasilinearization method with initial time difference simulation. Int J Electr Electron Phys Syst. 24(5) (2002)

3. Yakar, C, Yakar, A: An extension of the quasilinearization method with initial time difference. Dynamics of continuous, discrete and impulsive systems. Ser A Math Anal. 14(S2), 275-279 (2007)

4. Yakar, C, Yakar, A: Further generalization of quasilinearization method with initial time difference. J Appl Funct Anal. 4(4), 714-727 (2009)

5. Maleknejad, K, Najafi, E: Numerical solution of nonlinear volterra integral equations using the idea of quasilinearization. Commun Nonlinear Sci Numer Simulat. 16, 93-100 (2011). doi:10.1016/j.cnsns.2010.04.002

6. Amstera, $\mathrm{Pb}$, De Nápolia, P: A quasilinearization method for elliptic problems with a nonlinear boundary condition. Nonlinear Anal. 66, 2255-2263 (2007). doi:10.1016/j.na.2006.03.016

7. Kriveca, R, Mandelzweig, VB: Quasilinearization method and WKB. Comput Phys Commun. 174, 119-126 (2006) doi:10.1016/j.cpc.2004.12.017

8. Hristova, SG, Vatsala, AS: Quasilinearization for the periodic boundary value problem for systems of impulsive differential equations. J Appl Math Stochastic Anal 2006, 25 (2006). Article ID 98318

9. Oldham, KB, Spanier, J: The Fractional Calculus. Academic Press, New York (1974)

10. Samko, S, Kilbas, A, Marichev, O: Fractional Integrals and Derivatives: Theory and Applications. Gordon and Breach, New York. 1006 (1993)

11. Metzler, R, Schick, W, Kilian, HG, Nonnenmacher, TF: Relaxation in filled polymers: a fractional calculus approach. J Chem Phys. 103(16), 7180-7186 (1995). doi:10.1063/1.470346

12. Hilfe R (ed.): Applications of Fractional Calculus in Physics. World Scientific, River Edge. (2000)

13. Kiryakova, V: Generalized fractional calculus and applications. In Pitman Research Notes Mathematics Series, vol. 301, Longman-Wiley, New York (1994) 
14. Glockle, WG, Nonnenmacher, TF: A fractional calculus approach to self-similar protein dynamics. Biophys J. 68(1), 46-53 (1995). doi:10.1016/S0006-3495(95)80157-8

15. Podlubny, I: Fractional Differential Equations. Academic Press, San Diego (1999)

16. Lakshmikantham, V, Leela, S, Devi Vasundhara, J: Theory of Fractional Dynamic Systems. Cambridge Academic Publishers, Cambridge (2009)

17. Yakar, A: Some generalizations of comparison results for fractional differential equations. Comput Math Appl. 62, 3215-3220 (2011). doi:10.1016/j.camwa.2011.08.035

18. Yakar, A, Koksal, ME: Existence results for solutions of nonlinear fractional differential equations. Abst Appl Anal 2012, 12 (2012). Article ID 267108. doi:10.1155/2012/267108

19. Vasundhar Devi, J, Suseela, Ch: Quasilinearization for Caputo fractional differential equations. Commun Appl Anal. 12(4), 407-418 (2008)

20. Vasundhara Devi, J, Mc Rae, FA, Drici, Z: Generalized quasilinearization for Caputo fractional differential equations. Comput Math Appl. 59(3), 1057-1062 (2010). doi:10.1016/j.camwa.2009.05.017

21. Yakar, C, Yakar, A: A refinement of quasilinearization method for Caputo's sense fractional order differential equations. Abst Appl Anal 2010 (2010). Article ID 704367. doi:10.1155/2010/704367

22. Yakar, A: Method of Quasilinearization for Nonlinear Fractional Order Differential Equations. PhD Thesis, Gebze Institute of Technology. (2010)

23. Denton, Z, Vatsala, AS: Fractional integral inequalities and applications. Comput Math Appl. 59, 1087-1094 (2010). doi:10.1016/j.camwa.2009.05.012

24. Vasundhara Devi, J, Mc Rae, FA, Drici, Z: Variational Lyapunov method for fractional differential equations. Comput Math Appl (2012). doi:10.1016/j.camwa.2012.01.070

doi:10.1186/1687-1847-2012-92

Cite this article as: Yakar: Initial time difference quasilinearization for Caputo Fractional Differential Equations. Advances in Difference Equations 2012 2012:92.

\section{Submit your manuscript to a SpringerOpen ${ }^{\odot}$} journal and benefit from:

- Convenient online submission

- Rigorous peer review

- Immediate publication on acceptance

- Open access: articles freely available online

- High visibility within the field

- Retaining the copyright to your article

Submit your next manuscript at $\boldsymbol{s p r i n g e r o p e n . c o m ~}$ 